\title{
Composição química e energética de alguns alimentos para frangos de corte em duas idades
}

\author{
Rafaela Antonia Ramos Generoso ${ }^{1}$, Paulo Cezar Gomes ${ }^{2}$, Horacio Santiago Rostagno ${ }^{2}$, Luiz \\ Fernando Teixeira Albino ${ }^{2}$, Sergio Luiz de Toledo Barreto ${ }^{2}$, Gladstone Brumano ${ }^{1}$
}

\footnotetext{
1 Pós-graduação em Zootecnia - UFV.

2 Departamento de Zootecnia - UFV.
}

RESUMO - Este experimento foi conduzido com a finalidade de determinar os valores de energia metabolizável aparente (EMA) e energia metabolizável aparente corrigida pelo balanço de nitrogênio (EMA ${ }_{n}$ ) e analisar a composição química de alguns alimentos. Utilizou-se o método tradicional de coleta total de excretas com pintos de corte machos nos períodos de 21 a 30 dias e de 41 a 50 dias de idade. As aves foram distribuídas em delineamento inteiramente casualizado, com 11 dietas, 10 com alimentos-teste e uma dieta-referência), cada uma com seis repetições de seis e quatro aves por unidade experimental para o primeiro e o segundo período, respectivamente. Os alimentos estudados foram: quirera de arroz; farelo de arroz integral; milho; sorgo; farelo de trigo; farelo de soja; farelo de amendoim; farelo de algodão 28\% PB; e duas leveduras de cana-de-açúcar (40 e $43 \% \mathrm{~PB}$ ). Os valores de $\mathrm{EMA}_{\mathrm{n}}$ (kcal/kg matéria natural, $\mathrm{MN}$ ), no primeiro e segundo períodos experimentais foram, respectivamente: para levedura 40\%PB: 2.395 e 2.483; para levedura 43\%PB: 2.626 e 2.726; para o farelo de soja: 2.202 e 2.306; para farelo de algodão 28\%: 1.605 e 1.786; para farelo de amendoim: 2.155 e 2.471; para farelo de trigo: 1.683 e 1.877 ; para sorgo: 3.165 e 3.364; para milho: 3.351 e 3.524; para farelo de arroz integral: 2.446 e 2.650; e para a quirera de arroz: 3.138 e 3.278. Os valores EMA e EMA , de todos os alimentos, foram superiores no segundo período experimental, indicando que, com o avançar da idade, as aves melhoram o aproveitamento dos alimentos.

Palavras-chave: coleta total, frango de corte, ingrediente, método tradicional

\section{Chemical and energy composition of some feeds for broiler chicks and two ages}

\begin{abstract}
This experiment was carried out to determine of the values of apparent metabolizable energy (AME) and nitrogen corrected apparent metabolizable energy (AMEn) and to determine of the chemical composition of some feeds. The traditional method of total excreta collection was used with male broilers chickens in the period from 21 to 30 days old and 41 to 50 days old. The birds were distributed to a completely randomized experimental design, with eleven diets, ten with tested feeds and one reference diet, and each diet with six replications and six and four birds per experimental unit. The following feeds were studied: broken rice, whole rice meal, corn, sorghum, wheat bran, soybean meal, peanut meal, and cottonseed meal, $28 \% \mathrm{CP}$, and two sugarcane yeast (40 and 43\% CP). The values of AMEn (kcal/kg as fed), in the first and second experimental periods were, respectively, for sugarcane yeast $40 \%$ CP: 2.395 and 2.483, for sugarcane yeast 43\% CP: 2.626 and 2.726 , for soybean meal: 2.202 and 2.306, for cottonseed meal 28\% CP: 1.605 and 1.786: for peanut meal: 2.155 and 2.471, for wheat bran: 1.683 e 1.877, for sorghum: 3.165 and 3.364, for corn: 3.351 and 3.524, for whole rice meal: 2.446 and 2.650 and for the broken rice: 3.138 and 3.278. All values of AME and AMEn, for al feeds, were higher in second experimental period, showing that, with advancing of the age, birds improve the utilization of feeds.
\end{abstract}

Key Words: broiler chicks, ingredient, total collection, traditional method

\section{Introdução}

Com o aumento dos custos de produção e/ou a melhoria no potencial genético das aves, nutricionistas buscam alternativas que atendam às exigências dos animais nas suas diferentes fases de produção. A utilização de alimentos alternativos e de subprodutos da indústria é economicamente interessante para a pro- dução animal. Todavia, para a formulação de rações nutricionalmente viáveis, é importante conhecer o valor nutritivo dos alimentos, o que torna necessário determinar a composição química, a disponibilidade dos nutrientes, a concentração e a disponibilidade de energia dos alimentos.

A variação na composição química dos alimentos disponíveis no Brasil, decorrente de diversos fatores, é 
um problema para os nutricionistas. Assim, trabalhos têm sido desenvolvidos com os objetivos de atualizar os valores nutricionais dos alimentos comumente utilizados em rações para aves e conhecer o valor nutritivo de novos alimentos, o que torna as tabelas mais completas e com valores mais precisos (Carvalho, 2004; Nery, 2005; Brumano, 2006).

A determinação dos valores de energia metabolizável (EM) dos alimentos é fundamental, pois é a forma mais utilizada no cálculo de rações para aves. A precisão desses valores e sua correta utilização são necessárias para se obter ótima produtividade e máxima rentabilidade.

Este trabalho foi realizado com o objetivo de determinar a composição química e os valores de energia metabolizável aparente e energia metabolizável aparente corrigida de alguns alimentos utilizados na formulação de rações para frangos de corte em diversas idades.

\section{Material e Métodos}

O experimento foi realizado no setor de avicultura do Departamento de Zootecnia da Universidade Federal de Viçosa e as análises químicas, no Laboratório de Nutrição Animal do Departamento de Zootecnia da Universidade Federal de Viçosa. Os teores de matéria seca (MS), proteína bruta (PB), extrato etéreo (EE), energia bruta (EB), fibra bruta (FB), fibra em detergente neutro (FDN), fibra em detergente ácido (FDA), matéria mineral (MM), cálcio (Ca) e fósforo (P) dos alimentos foram determinados conforme as técnicas descritas por Silva (2002).

Os alimentos testados foram: quirera de arroz; farelo de arroz integral; milho; sorgo; farelo de trigo; farelo de soja; farelo de algodão; farelo de amendoim e duas leveduras (com 40 ou $43 \%$ de PB).

Os valores de energia metabolizável aparente (EMA) e energia metabolizável aparente corrigida (EMAn) foram determinados utilizando-se o método tradicional de coleta total de excretas e pintos de corte em duas idades (de 21 a 30 e de 41 a 50 dias de idade). Utilizaram-se 396 pintos machos da linhagem Ross mantidos em círculos de proteção até aos 21 dias de idade recebendo ração inicial para frangos de corte. Aos 21 dias de idade, as aves foram pesadas e selecionadas de acordo com o peso inicial médio $(725,05 \mathrm{~g})$ e posteriormente foram transferidas para gaiolas de estudos de metabolismo, dispostas em esquema de bateria de dois andares, onde foram mantidas até os 31 dias de idade. As temperaturas mínima e máxima, médias, registradas durante o período experimental foram de $18 \mathrm{e} 24^{\circ} \mathrm{C}$, respectivamente.
Utilizou-se no primeiro experimento um delineamento experimental inteiramente casualizado (DIC), com 11 tratamentos (dez alimentos e uma dieta-referência), cada um com seis repetições e seis aves por unidade experimental. Entre os alimentos testados, cinco (duas leveduras, farelo de soja, farelo de algodão, farelo de amendoim) substituíram 20\% da dieta-referência e os demais (farelo de trigo, sorgo, milho, farelo de arroz integral, quirera de arroz) substituíram 30\% da dieta-referência (Tabela 1).

As dietas foram fornecidas à vontade durante dez dias: cinco de adaptação e cinco de coleta total das excretas, realizada em intervalos de 12 horas. As bandejas utilizadas na coleta das excretas foram revestidas com plástico, sob o piso de cada unidade experimental, de acordo com metodologia descrita por Sibbald (1976).

Ao final do experimento, determinou-se a quantidade total de ração consumida por repetição. As excretas coletadas foram acondicionadas em sacos plásticos, que foram devidamente identificados, pesados e armazenados em freezer até o final do período de coleta. Posteriormente, as excretas foram descongeladas, homogeneizadas e amostradas. As amostras foram pré-secas em estufa de ventilação forçada a $60^{\circ} \mathrm{C}$ durante 72 horas e submetidas a análises laboratoriais para determinação dos teores de MS, N e EB.

Aos 31 dias de idade, as aves foram conduzidas novamente ao galpão de alvenaria, onde foram alimentadas durante dez dias com ração de crescimento para frangos de corte, segundo recomendações de Rostagno et al. (2000).

Aos 41 dias de idade, as aves foram novamente mantidas nas gaiolas para início do segundo período experimental, quando as 264 aves que estavam com peso inicial médio de $2.100 \mathrm{~g}$ foram distribuídas em delineamento inteiramente casualizado, com 11 tratamentos (dez alimentos e uma dieta-referência), cada um com seis repetições e quatro aves por unidade experimental. As temperaturas mínima e máxima, médias, registradas durante este período experimental foram de 25 e $29^{\circ} \mathrm{C}$, respectivamente.

Nesta fase, utilizou-se a mesma metodologia da fase anterior, incluindo a dieta-referência, as dietas-teste, o método de coleta de excretas das aves e as análises laboratoriais.

Uma vez obtidos os resultados das análises laboratoriais dos alimentos, da dieta-referência, das dietasteste e das excretas, foram calculados os valores de EMA e EMA $\mathrm{n}$ utilizando-se equações propostas por Matterson et al. (1965). 
Tabela 1 - Composição da dieta-referência (MN)

\begin{tabular}{lc}
\hline Item & $\%$ \\
\hline Ingrediente & \\
Milho & 56,35 \\
Farelo de soja & 35,82 \\
Óleo de soja & 3,85 \\
Fosfato bicálcio & 1,82 \\
Calcário calcítico & 0,98 \\
Sal & 0,46 \\
DL-metionina (99\%) & 0,24 \\
L-lisina HCL (98\%) & 0,16 \\
Mistura vitamínica1 & 0,10 \\
Mistura mineral' & 0,05 \\
Anticoccidoano & 0,05 \\
Cloreto de colina 60\% & 0,10 \\
Avilamicina 10\% & 0,01 \\
Antioxidante BHT & 0,01 \\
\hline Composição calculada & \\
\hline Proteína bruta (\%) & 21,00 \\
Energia metabolizável (kcal/kg) & 3.050 \\
Cálcio (\%) & 0,960 \\
Fósforo disponível (\%) & 0,450 \\
Sódio (\%) & 0,222 \\
Metionina + cistina (\%) & 0,897 \\
Metionina + cistina digestível (\%) & 0,816 \\
Lisina (\%) & 1,263 \\
Lisina digestível (\%) & 1,145 \\
Treonina (\%) & 0,824 \\
Treonina digestível (\%) & 0,717 \\
Triptofano (\%) & 0,266 \\
Triptofano digestível (\%) & 0,241 \\
\hline 1 Rovimix (Roche) - Níveis de garantia por quilo do produto: vit. A - \\
10.000.000 Ul; vit. D3 - 2.000.000 Ul; vit. E - 30.000 Ul; vit. B1 - 2,0 g; \\
vit. B6 - 4,0 g; ácido pantotênico - 12,0 g; biotina - 0,10 g; vit. K3 - 3,0 \\
g; ácido fólico - 1,0 g; ácido nicotínico - 50,0 g; vit. B12 - 15.000 mcg; \\
25e - 0, 25 g; e Veículo q.s.p - 1.000 g. \\
Roligomix (Roche). Níveis de garantia por quilo do produto: Mn - 16,0 g; \\
- 1.000 g. g; Zn - 100,0 g; Cu - 20,0 g; Co - 2,0 g; I - 2,0 g; veículo q.s.p. \\
\hline
\end{tabular}

\section{Resultados e Discussão}

Os valores da composição química dos alimentos estudados (Tabela 2) diferiram tanto dos resultados da literatura nacional (Rostagno et al., 2000; Rodrigues, 2000; D’Agostini, 2001; Brumano, 2006; Nery, 2005; Rostagno et al., 2005) quanto da literatura internacional (Janssen, 1989; NRC 1994; Dale, 1999; FEDNA, 2003). Todavia, estas diferenças eram esperadas, pois existem variações nas condições de solo, clima e entre cultivares. Além disso, os subprodutos são obtidos em diversas condições de processamento e manejo, que podem afetar sua composição.

A composição química da quirera de arroz foi similar à descrita por Rostagno et al. (2005) e diferiu apenas quanto ao teor de EE, que foi 3,28\% inferior ao citado por esses autores, fato que justifica o valor de EB encontrado. Os valores de composição química, principalmente de EB, foram inferiores aos descritos na tabela da EMBRAPA (1991).

A composição química do farelo de arroz integral, quando comparada à descrita nas tabelas nacionais (EMBRAPA, 1991; Rostagno et al., 2005), apresentou maior variação quanto aos teores de EE, que foram 20,8\% inferiores ao encontrado por Rostagno et al. (2005) e $27,77 \%$ menores que o citado por Pupa (1995), o que confirma os valores inferiores de energia bruta. Os valores de fibra bruta foram $41,69 \%$ inferiores aos descritos por Pupa (1995).

Os valores de EE e EB obtidos nos farelos de algodão e no farelo de amendoim foram próximos aos citados por Rostagno et al. (2005) e inferiores aos encontrados por FEDNA (2003). Os valores de EB encontrados para estes alimentos foram diretamente proporcionais aos teores de EE contidos nos mesmos.

A composição química das leveduras apresentou grande variação se comparada aos valores descritos na tabela da EMBRAPA (1991). Os resultados, com exceção do teor de fósforo, foram inferiores aos relatados nesta tabela, contudo, estiveram em conformidade com os citados por Rostagno et al. (2005).

Tabela 2 - Composição química e energética dos alimentos (MN) ${ }^{1}$

\begin{tabular}{lcccccccccc}
\hline Alimento & ${ }^{2} \mathrm{MS} \%$ & ${ }^{3} \mathrm{~PB} \%$ & ${ }^{2} \mathrm{EE} \%$ & ${ }^{2} \mathrm{FB} \%$ & ${ }^{2} \mathrm{FDN} \%$ & ${ }^{2} \mathrm{FDA} \% \mathrm{rf}$ & ${ }^{2} \mathrm{MM} \%$ & ${ }^{2} \mathrm{Ca} \%$ & ${ }^{2} \mathrm{P} \%$ & ${ }^{2} \mathrm{~EB}(\mathrm{kcal} / \mathrm{kg})$ \\
\hline Quirera de arroz & 86,96 & 8,40 & 1,18 & 0,42 & 5,10 & 8,25 & 0,80 & 0,06 & 0,17 & 3.756 \\
Farelo de arroz integral & 87,25 & 12,34 & 11,73 & 7,23 & 22,77 & 14,28 & 10,44 & 0,13 & 1,70 & 4.007 \\
Farelo de algodão 28\% & 88,99 & 28,29 & 1,15 & 22,34 & 41,37 & 29,44 & 4,35 & 0,19 & 0,76 & 4.029 \\
Farelo de amendoim & 88,89 & 45,06 & 0,98 & 6,97 & 16,20 & 12,21 & 6,29 & 0,16 & 0,58 & 4.328 \\
Levedura 40\% & 89,99 & 38,83 & 0,45 & 0,46 & 1,09 & 0,86 & 3,11 & 0,25 & 0,76 & 4.225 \\
Levedura 43\% & 90,55 & 41,23 & 0,48 & 0,48 & 1,00 & 0,96 & 3,25 & 0,27 & 0,79 & 4.255 \\
Milho & 87,04 & 8,00 & 3,78 & 1,59 & 14,64 & 4,11 & 1,18 & 0,03 & 0,23 & 3.898 \\
Farelo de soja & 87,96 & 44,37 & 1,58 & 5,25 & 14,75 & 8,60 & 5,82 & 0,22 & 0,49 & 4.050 \\
Sorgo & 86,95 & 8,81 & 3,04 & 2,80 & 12,22 & 6,09 & 1,15 & 0,02 & 0,22 & 3.910 \\
Farelo de trigo & 87,05 & 15,12 & 3,54 & 8,90 & 38,38 & 12,80 & 4,35 & 0,12 & 0,90 & 3.892 \\
\hline
\end{tabular}

${ }^{1}$ Análises realizadas no Laboratório de Nutrição Animal do Departamento de Zootecnia da Universidade Federal de Viçosa.

2 MS - matéria seca; PB - proteína bruta; EE - extrato etéreo; FB - fibra bruta; FDN - fibra detergente neutro; FDA - fibra detergente acido; MM - matéria mineral; Ca - cálcio; P - fósforo; EB - energia bruta.

${ }^{3}$ Laboratório da Degussa, ${ }^{3}$ Lab Degussa. 
Os resultados das análises de composição química do milho avaliados neste trabalho foram semelhantes aos encontrados por Rostagno et al. (2005), Fischer (1998), Pupa (1995) e EMBRAPA (1991), exceto os de FB e EB, que foram inferiores aos encontrados por D'Agostini (2001).

A composição química em EE e EB do farelo de soja foi ligeiramente inferior àquela citada por Rostagno et al (2005), Pupa (1995) e EMBRAPA (1991). Os valores de PB e EE determinados no sorgo foram similares aos descritos por Rostagno et al. (2005), FEDNA (2003) e Pupa (1995). No entanto, os valores de PB foram 10,33\% superiores aos encontrados por Nunes (2001) e 13,73\% aos publicados pela EMBRAPA (1991). Os valores de EE foram superiores aos citados por Nunes (2001), Fischer (1998) e Pupa (1995), o que ocasionou maiores valores de EB em comparação aos obtidos por esses autores.

A composição química do farelo de trigo foi similar à encontrada por Nunes (2001) e Rostagno et al. (2005), que foram inferiores aos citados pela EMBRAPA (1991).

Os valores de EMA e EMA $A_{n}$ diferiram entre os alimentos estudados (Tabela 3). Os de EMA foram superiores

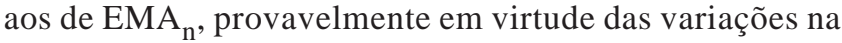
composição química dos alimentos. Este resultado era esperado, visto que os valores de EM foram determinados com aves em crescimento e nesta fase ocorre maior retenção de nitrogênio para deposição de tecido protéico, que é mais acentuada quando se faz correção pelas perdas endógenas e metabólicas.

Segundo Wolynetz \& Sibbald (1984), em condições de consumo à vontade, os valores de EMA são superiores aos de EMAn, quando a retenção de nitrogênio é positiva. Como nesta pesquisa as aves apresentaram consumo normal de ração (à vontade), o valor de nitrogênio retido foi maior que zero e, conseqüentemente, a EMA superou os valores de $\mathrm{EMA}_{\mathrm{n}}$.

Os valores de EMA dos alimentos energéticos variaram de 1.820 a $3.368 \mathrm{kcal} / \mathrm{kg}$ e os de EMA , de 1.683 a $3.351 \mathrm{kcal} / \mathrm{kg}$ de matéria seca. Os alimentos protéicos variaram de 1.625 a $2.724 \mathrm{kcal} / \mathrm{kg}$ para EMA e os de 1.605 a $2.626 \mathrm{kcal} / \mathrm{kg}$ de matéria seca para $\mathrm{EMA}_{\mathrm{n}}$.

Na segunda fase avaliada, os valores de EMA e de EMA $_{n}$ foram superiores $(\mathrm{P}<0,01)$ aos obtidos na primeira fase para todos os alimentos. Para a quirera de arroz, os

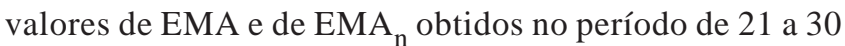
dias de idade foram aproximadamente $5 \%$ inferiores aos encontrados por Rostagno et al. (2005).

Nas aves mais jovens, os valores de EMA e EMA determinados para o farelo de arroz integral foram 3 e $2 \%$ inferiores, respectivamente, aos citados por Rostagno et al. (2005) e pela tabela da EMBRAPA (1991). Essa variação pode ser explicada pela maior proporção de casca no farelo, o que contribui para aumentar o teor de FB. Além disso, o farelo utilizado apresentou valores de EE inferiores aos encontrados por esses autores.

$\mathrm{O}$ valor de $\mathrm{EMA}_{\mathrm{n}}$ encontrado para o farelo de algodão no primeiro período experimental, $1.605 \mathrm{kcal} / \mathrm{kg}$, foi semelhante ao determinado por Rostagno et al. (2005), $1.666 \mathrm{kcal} / \mathrm{kg}$. Entretanto, foi superior ao descrito por FEDNA (2003), $1520 \mathrm{kcal} / \mathrm{kg}$ e inferior ao obtido por Brumano (2006), $1.963 \mathrm{kcal} / \mathrm{kg}$. No entanto, o valor de EMA do farelo de algodão nessa fase, $1,625 \mathrm{kcal} / \mathrm{kg}$, foi inferior ao encontrado por Brumano (2006), de $1.965 \mathrm{kcal} / \mathrm{kg}$.

A diferença encontrada nas aves de 41 a 50 dias de idade $(\mathrm{P}<0,05)$ para os alimentos que possuem elevado teor de fibra pode estar associada ao desenvolvimento fisiológico do trato digestório com o avançar da idade, que acarreta melhor aproveitamento da fibra.

Os valores de $\mathrm{EMA}_{\mathrm{n}}$ obtidos para o farelo de amendoim no período de 21 a 30 dias de idade foram 3,7\% inferiores aos descritos por Rostagno et al. (2005). Todavia, foram 5,6\% superiores aos citados na tabela da EMBRAPA (1991).

A levedura com $40 \%$ de proteína bruta apresentou no período de 21 a 30 dias de idade dos frangos de corte valor de EMAn de $2.395 \mathrm{kcal} / \mathrm{kg}$, valor inferior aos descritos pela EMBRAPA (1991), de 2.947 kcal/kg, e Rostagno et al.(2005), $2.506 \mathrm{kcal} / \mathrm{kg}$. A levedura contendo 43\% de proteína bruta, no entanto, apresentou $2.626 \mathrm{kcal} / \mathrm{kg}$ de EMAn, valor superior aos reportados pelo NRC (1994), $2.160 \mathrm{kcal} / \mathrm{kg}$, e por Rostagno et al. (2005), $2.506 \mathrm{kcal} / \mathrm{kg}$, e inferior apenas aos valores da EMBRAPA (1991), $2.947 \mathrm{kcal} / \mathrm{kg}$.

Os valores encontrados no segundo período experimental foram superiores aos do primeiro período, o que pode ser explicado pelo fato de as leveduras serem um alimento de granulometria fina e poderem provocar irritabilidade na mucosa da ave mais jovem. Além disso, o trato digestório da ave mais velha pode eliminar de forma mais eficiente o excesso de proteína contido neste alimento.

No período de 21 a 30 dias, o valor de EMA $\mathrm{n}_{\mathrm{n}}$ encontrado para o milho (3.351 kcal/kg) foi inferior aos citados por Rostagno et al. (2005), pela tabela da EMBRAPA (1991) e por Rodrigues (2001), de 3.381, 3.876 e 3.699 kcal/kg, respectivamente, contudo, foram superiores aos descritos por FEDNA (2003), $3.260 \mathrm{kcal} / \mathrm{kg}$. Os valores de EMA (3368 kcal $/ \mathrm{kg}$ ) foram inferiores aos encontrados por Borges (2001), 3.749 kcal/kg, e D’ Agostini (2004), 3.246 kcal/kg.

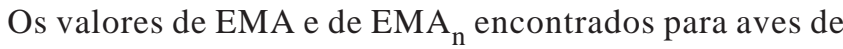
41 a 50 dias de idade, 3.547 e $3.524 \mathrm{kcal} / \mathrm{kg}$, respectivamente, foram semelhantes aos encontrados nas aves de 21 a 30 dias de idade. 
Tabela 3 - Valores de energia metabolizável aparente (EMA) e aparente corrigida (EMAn) dos alimentos em aves de 21 a 30 dias e de 41 a 50 dias, na matéria natural ${ }^{1}$

\begin{tabular}{|c|c|c|c|c|}
\hline \multirow{2}{*}{ Alimento } & \multicolumn{2}{|c|}{ EMA (kcal/kg) } & \multicolumn{2}{|c|}{ EMAn (kcal/kg) } \\
\hline & 21 a 30 dias & 41 a 50 dias & 21 a 30 dias & 41 a 50 dias \\
\hline Quirera de arroz & 3.155 & 3.293 & 3.138 & 3.278 \\
\hline Farelo de arroz integral & 2.458 & 2.660 & 2.446 & 2.650 \\
\hline Farelo de algodão $28 \%$ & 1.625 & 1.786 & 1.605 & 1.734 \\
\hline Farelo de amendoim & 2.194 & 2.555 & 2.155 & 2.471 \\
\hline Levedura $40 \%$ & 2.497 & 2.570 & 2.395 & 2.483 \\
\hline Levedura $43 \%$ & 2.724 & 2.846 & 2.626 & 2.726 \\
\hline Milho & 3.368 & 3.547 & 3.351 & 3.524 \\
\hline Farelo de soja & 2.251 & 2.359 & 2.202 & 2.306 \\
\hline Sorgo & 3.175 & 3.396 & 3.165 & 3.374 \\
\hline Farelo de trigo & 1.820 & 2.019 & 1.683 & 1.877 \\
\hline Média & $2.527 b$ & $2.703 a$ & $2.477 \mathrm{~B}$ & $2.642 \mathrm{~A}$ \\
\hline
\end{tabular}

${ }_{1}^{1}$ Médias seguidas de letras iguais nas linhas não diferem $(P>0,01)$ pelo teste Tukey.

O valor de EMA $\mathrm{n}$ obtido para o farelo de soja em aves mais jovens foi de $2.202 \mathrm{kcal} / \mathrm{kg}$, semelhante ao descrito por Rostagno et al. (2005), $2.256 \mathrm{kcal} / \mathrm{kg}$, entretanto, foi superior ao citado na FEDNA (2003), $2.180 \mathrm{kcal} / \mathrm{kg}$, e inferior ao apresentado pelo NRC (1994), de $2.230 \mathrm{kcal} / \mathrm{kg}$.

Os resultados de EMA e EMAn obtidos com o sorgo nas duas fases avaliadas foram, respectivamente, 3.175 e $3.396 \mathrm{kcal} / \mathrm{kg}$ na fase de 21 a 30 dias e de 3.165 e $3.374 \mathrm{kcal} / \mathrm{kg}$ na fase de 41 a 50 dias de idade. O resultado de EMAn na fase de 21 a 30 dias, $3.165 \mathrm{kcal} / \mathrm{kg}$, foi inferior ao descrito pela EMBRAPA (1991), $3.290 \mathrm{kcal} / \mathrm{kg}$, pelo NRC (1994), 3.288 kcal/kg, e por Rostagno et al. (2005), 3.192 kcal/kg. Contudo, foram superiores aos relatados por FEDNA (2003) para esta fase, de $3.060 \mathrm{kcal} / \mathrm{kg}$.

O farelo de trigo avaliado nas aves de 21 a 30 dias de idade apresentou valor de $\mathrm{EMA}_{\mathrm{n}}$ de $1.683 \mathrm{kcal} / \mathrm{kg}$, superior ao descrito na tabela da EMBRAPA (1991), de $1.590 \mathrm{kcal} / \mathrm{kg}$. No entanto, foram inferiores aos descritos por Nunes (2001), $1.864 \mathrm{kcal} / \mathrm{kg}$, e Rostagno et al. (2005), $1.824 \mathrm{kcal} / \mathrm{kg}$. A EMA encontrada para o farelo de trigo no período de 21 a 30 dias, $1.820 \mathrm{kcal} / \mathrm{kg}$, foi inferior à encontrada por Nunes (2001), $1.940 \mathrm{kcal} / \mathrm{kg}$. Os valores de EMA (2019 kcal/kg) e de $\operatorname{EMA}_{\mathrm{n}}(1.877 \mathrm{kcal} / \mathrm{kg})$ encontrados nas aves de 41 a 50 dias de idade foram superiores aos obtidos nas aves de 21 a 30 dias de idade, provavelmente em virtude do melhor aproveitamento da fibra desse farelo pelas aves mais velhas.

De modo geral, os valores de EMA e de $\mathrm{EMA}_{\mathrm{n}}$ de todos os alimentos estudados foram maiores nas aves na fase de 41 a 50 dias de idade $(\mathrm{P}<0,01)$. Batal \& Parsons (2002) relataram que a idade das aves interfere nos valores de EMA e EMA ${ }_{\mathrm{n}}$ e na digestibilidade aparente de vários nutrientes da dieta.
Assim, é necessário aprofundar os estudos sobre a composição química e os valores de energia metabolizável aparente e energia metabolizável aparente corrigida de alguns alimentos utilizados na formulação de rações para frangos de corte visando complementar as tabelas nacionais e internacionais com valores energéticos de acordo com idade das aves para que a formulação de rações seja otimizada.

\section{Conclusões}

Os valores de $\mathrm{EMA}_{\mathrm{n}}(\mathrm{kcal} / \mathrm{kg})$, na matéria natural, para as aves de 21 a 30 dias e de 41 a 50 dias de idade foram, respectivamente: para quirera de arroz: 3.138 e 3.278; para farelo de arroz integral: 2.446 e 2.650; para farelo de algodão 28\% de PB: 1.605 e 1.786; para farelo de amendoim: 2.155 e 2.471; para levedura $40 \%$ de $\mathrm{PB}$ : 2.395 e 2.483 ; para levedura 43\% de PB: 2.626 e 2.726; para milho: 3.351 e 3.524; para farelo de soja: 2.020 e 2.306; para sorgo: 3.165 e 3.374; para farelo de trigo: 1.683 e 1.877. Os valores de EMA e EMA $_{n}$ de todos os alimentos obtidos em aves mais velhas foram superiores $(\mathrm{P}<0,01)$ aos encontrados em aves mais novas, uma vez que aves mais velhas possuem aparelho digestivo mais desenvolvido, portanto, aproveitam melhor os alimentos.

\section{Literatura Citada}

BATAL, A.B; PARSONS, C.M. Effects of age on nutrient digestibility in chicks fed different diets. Poultry Science, v.81, p.400-407. 2002.

BRUMANO, G.; GOMES, P.C.; ALBINO, L.F.T. et al. Composição química e valores de energia metabolizável de alimentos protéicos determinados com frangos de corte em diferentes idades. Revista Brasileira de Zootecnia, v.35, n.6, p.2297-2302, 2006. 
CARVALHO, D.C.O.; ALBINO, L.F.T.; ROSTAGNO, H.S. et al. Composição química e energética de amostras de milho submetidas a diferentes temperaturas de secagem e períodos de armazenamento. Revista Brasileira de Zootecnia, v.33, n.2, p.358-364, 2004.

D’AGOSTINI, P.; GOMES, P.C.; ALBINO, L.F.T. et al Coeficientes de digestibilidade verdadeira de alguns alimentos determinados com galos cecectomizados. In: REUNIÃO ANUAL DA SOCIEDADE BRASILEIRA DE ZOOTECNIA, 38., 2001. Piracicaba. Anais... Piracicaba: Sociedade Brasileira de Zootecnia, 2001. v.1, p.795-796.

D’AGOSTINI, P.; GOMES, P.C.; ALBINO, L.F.T. et al. Valores de composição química e energética de alguns alimentos para aves. Revista da Sociedade Brasileira de Zootecnia, v.33, n.1, p.128-134, 2004.

DALE, N. Ingredient analysis table: 1999 Edition. Feedstuffs, v.71, n.31, p.24-31, 1999.

EMPRESA BRASILEIRA DE PESQUISA AGROPECUÁRIA EMBRAPA. Tabela de composição química e valores energéticos de alimentos para suínos e aves. 3.ed. Concórdia: CNPSA, 1991, 97p. [Documento 19].

FUNDACIÓN ESPAÑOLA PARA EL DESARROLLO DE LA NUTRICIÓN ANIMAL - FEDNA. Tablas fedna de composición y valor nutritivo de alimentos para la fabricación de piensos compuestos. 2.ed. Madrid: 2003. 253p.

FISCHER JR., A.A.; ALBINO, L.F.T.; ROSTAGNO, H.S. et al. Determinação dos valores de energia metabolizável de alguns alimentos usados na alimentação de aves. Revista da Sociedade Brasileira de Zootecnia, v.27, n.2, p.314-318,1998.

JANSSEN, W.M.M.A. European table of energy values for poultry feedstuffs. 3.ed. Beekbergen, 1989. 84p. (Spelderholt Center for Poultry Research and Information Services).

MATTERSON, L.D.;POTTER, L.M.; STUTZ, M.W. et al. The metabolizable energy of feed ingredients for chickens. University of Connecticut Storrs. Agricultural Experiment Station Research Report, v.11, 11p, 1965.

NATIONAL RESEARCH COUNCIL - NRC. Nutrient requirements of poultry. 9.ed. Washington, D.C.: National Academy of Sciences, 1994. 155p.
NERY, L.R. Valores de energia metabolizável e de aminoácidos digestíveis de alguns alimentos para aves. Viçosa, MG: Universidade Federal de Viçosa, 2005. 100p. Dissertação (Mestrado em Zootecnia) - Universidade Federal de Viçosa, 2005.

NUNES, R.V.; ROSTAGNO, H.S.; ALBINO, L.F.T. et al. Composição bromatológica, energia metabolizável e equações de predição da energia do grão e de subprodutos do trigo para pintos de corte. Revista Brasileira de Zootecnia, v.30, n.3, p.785-793, 2001.

PUPA, J.M.R. Rações para frangos de corte formuladas com valores de aminoácidos digestíveis verdadeiros, determinados com galos cecectomizados. Viçosa, MG: Universidade Federal de Viçosa, 1995. 63p. Dissertação (Mestrado em Zootecnia) - Universidade Federal de Viçosa, 1995.

RODRIGUES, P.B.; ROSTAGNO, H.S.; ALBINO, L.F.T et al. valores energéticos do milheto, do milho e subprodutos do milho, determinados com frangos de corte e galos adultos. Revista Brasileira de Zootecnia, v.30, n.6, p.1767-1778, 2001.

RODRIGUES, P.B. Valores energéticos da soja e subprodutos da soja, determinados com frangos de corte e galos adultos. Revista da Sociedade Brasileira de Zootecnia, v.31, n.4, p.17711782, 2002.

ROSTAGNO, H.S.; ALBINO, L.F.T.; DONZELE, J.L. et al. Tabelas brasileiras para aves e suínos: composição de alimentos e exigências nutricionais. Viçosa, MG: Editora UFV, 2000. 141p.

ROSTAGNO, H.S.; ALBINO, L.F.T.; DONZELE, J.L. et al. Tabelas brasileiras para aves e suínos: composição de alimentos e exigências nutricionais. 2.ed. Viçosa, MG: Editora UFV, 2005. $186 \mathrm{p}$.

SIBBALD, I.R. A bioassay for metabolizable energy in feedingstuffs. Poultry Science, v.55, n.1, p.303-308, 1976.

SILVA, D.J. Análise de alimentos (métodos químicos e biológicos). 2.ed. Viçosa: MG: Universidade Federal de Viçosa, 1990. 165p.

WOLYNETZ, M.N.; SIBBALD, I.R. Relationships between apparent na true metabolizable energy and the effects of a nitrogen correction. Poultry Science, v.63, n.7, p.13861399, 1984. 